\title{
CFD Investigation of Thermal and Pressurization Performation in LHe Tank
}

\author{
Wang Yuzhu ${ }^{\mathrm{a}}$, Cui Lande ${ }^{\mathrm{a}}$, Zhang Caigong ${ }^{\mathrm{b}}$, Kong Lingfen ${ }^{\mathrm{a}}$, Jia Wenlong ${ }^{\mathrm{b}}$ \\ ${ }^{a}$ Oil and Gas Engineering Research Institute, PetrochinaTarim Oilfield Company, Korla, Xinjiang,841000, \\ China \\ ${ }^{b}$ School of Petroleum Engineer, Southwest Petroleum University, Chengdu Sichuan 610500, China
}

Received: 27 September 2019; Accepted: 26 October 2019; Published: 08 February 2020

\begin{abstract}
Research on thermal response of liquid helium storage tank is an important part of non-destructive storage technology for liquid helium, However there are few reports on the thermal response of liquid helium storage tanks. Taking the thermal stratification, pressure rise phenomenon and natural convection of liquid helium storage tank as research objects, based on the Lee model, the finite volume method was used for the first time to study the non-steady-state thermal response which simultaneously considering the change of liquid helium and vapor helium properties. Thus the tank pressure, temperature and flow rate distribution at different times were obtained, and the effects of evaporation rate and filling rate on the pressure rise and temperature rise of the storage tank were analyzed. The research shows that with the increase of simulation time, the liquid helium shows thermal stratification. In addition, the pressure is distributed in a stepwise manner which is lower in the upper part, and higher in the lower part, while the pressure distribution in the gas phase space is more uniform; After the liquid helium in the near-wall area is heated, it rises along the wall surface to the free liquid surface under buoyancy lift, and then flows into the main flow area of the liquid helium; As the evaporation rate increases, the temperature rise and pressure rise rate in the gas phase space of the storage tank increase, while the filling rate have less influence on the temperature rise and pressure rise rate.This work provides guidance for non-destructive storage and transportation theory of liquid helium storage tanks.
\end{abstract}

Index Terms: Liquid helium, Storage tank, Thermal response, Numerical study.

(C) 2020 Published by MECS Publisher. Selection and/or peer review under responsibility of the Research Association of Mode rn Education and Computer Science

\footnotetext{
* Corresponding author.
}

E-mail address: 


\section{Introduction}

With the rapid development of aerospace, military industry and scientific research, the application of helium is becoming more and more important. Because helium resource is rare, cryogenic insulated storage tanks are currently mainly used for storage and transportation of liquid helium. But the boiling point of liquid helium is as low as $4.2 \mathrm{~K}$, resulting in heat leakage even if high-vacuum multi-layer insulation structure is adopted in liquid helium storage tanks. The heat leakage will cause liquid helium in the storage tank to evaporate at the phase interface after rising to saturation temperature, resulting in pressure rise in the gas zone of the storage tank, bringing hidden dangers to the safe storage and transportation of liquid helium. However, there are few reports on the thermal response research and the research is not comprehensive enough, which leads to the uncertainty of the thermal response discipline of the liquid helium storage tank. Therefore, it is necessary to research the thermal response phenomenon of liquid helium tanks.

At present, there are three methods for studying the thermal response of liquid helium storage tanks, which are theoretical methods, experimental methods, and numerical simulation methods. The theoretical methods have been in progress since 1950. Initially, liquid hydrogen, liquid, and liquid nitrogen were used as research objects. Later, due to the use of natural gas, the low-temperature storage of LNG was systematically studied. So far, the theory of cryogenic liquid storage is relatively mature. However the models used for liquid hydrogen, liquid nitrogen and LNG such as homogeneous surface evaporation model, saturated homogeneous model may no longer be applicable to liquid helium.

The experimental methods is the main research method for researching of thermal response discipline. However, the experimental method is expensive and there are few related research reports. There are supercritical helium is taken as the research object and the influence of daily evaporation rate and filling rate on the gas pressure of the storage tank is analyzed. The research shows that the gas phase pressure of the storage tank changes in a linear rule[1]. Moreover, there are pressure suppression experment on a 100L supercritical helium storage tank[2]. In the last century, some scholars used experimental methods to study the temperature distribution and pressure distribution of small liquid helium Dewar. The temperature distribution of liquid helium and gas helium in liquid helium Dewar has been experimentally studied, and drown the distribution curve of gas-phase temperature[3]. The 200L liquid helium Dewar has also been experimentally studied, so the temperature distribution discipline of helium in the neck tube of the storage tank has been obtained[4]. The 12L spherical liquid helium Dewar has been tested and studied to test the evaporation rate of liquid helium [5]. However, the above experimental research was carried out so early that no profound analysis of the distribution of the flow field, temperature field, and pressure field in the storage tank.

Computational Fluid Dynamics (CFD) is a convenient and low-cost research method[6], which has been widely used in the thermal response research of cryogenic liquids such as LNG, liquid nitrogen and liquid hydrogen. But there are no reports on numerical studies of the thermal response process of liquid helium storage tanks. Although the simulation results cannot completely match the actual thermal response of the liquid helium storage tank, however under the case that theoretical and experimental research cannot obtain comprehensive data on the thermal performance of the liquid helium storage tank,and under the circumstance that there are no article using numerical simulation methods to study the thermal response of liquid helium storage tanks, it is acceptable and meaningful to conduct a pilot research using CFD simulation technology to reveal the discipline of thermal response progess in liquid helium storage tank.

Based on this, considering the changes of physical parameters of liquid helium and gas helium, the thermal stratification, pressure rise discipline and natural convection of liquid helium storage tanks were studied using simulation methods. In this paper, the commercial software Fluent is adopted. 


\subsection{Physical model}

In this paper, the thermal stratification, pressure rise discipline and natural convectiont in $85 \mathrm{~m} 3$ horizontal railway liquid helium tanker is taken as the research object. And the structure of the tank is shown in Fig. 1. The inner container is made of $316 \mathrm{~L}$ stainless steel. The length of the inner container is $14 \mathrm{~m}$ (excluding the head), which is quite different from the inner diameter of the inner container of $2.8 \mathrm{~m}$ (diameter). The inner container is axisymmetric about the centerline of the cylinder. Therefore, the three-dimensional model is simplified to a two-dimensional circle with a radius of $1.4 \mathrm{~m}$ and the center is the origin of coordinates $(0,0)$. In order to analyze the temperature and pressure distribution of the flow field conveniently, an auxiliary analysis line as shown in Fig. 2 is added.

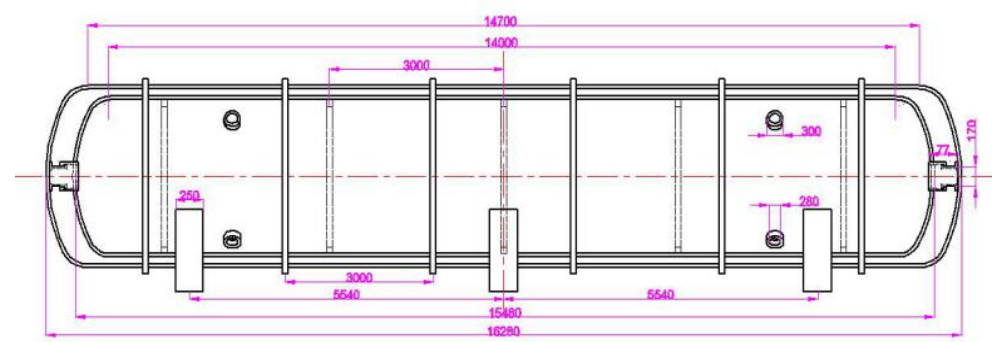

Fig.1 The geometric model of liquid helium tank

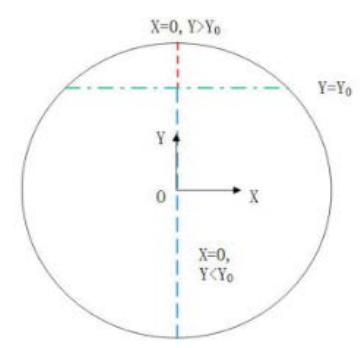

Fig.2 Added auxiliary analysis line

\subsection{Mathematical model}

$$
\begin{aligned}
& \frac{\partial \rho}{\partial t}+\sum_{i=1}^{3} \frac{\partial\left(\rho u_{i}\right)}{\partial x_{i}}=S_{m} \\
& \frac{\partial\left(\rho u_{i}\right)}{\partial t}+\frac{\partial\left(\rho u_{i} u_{j}\right)}{\partial x_{j}}=-\frac{\partial p}{\partial x_{i}}+\frac{\partial}{\partial x_{j}}\left[\mu\left(\frac{\partial u_{i}}{\partial x_{j}}+\frac{\partial u_{j}}{\partial x_{i}}\right)-\frac{2 \mu}{3} \frac{\partial u_{i}}{\partial x_{l}} \delta_{i j}\right]+\rho g_{i} \\
& \frac{\partial(\rho T)}{\partial t}+\operatorname{div}(\rho \vec{u} T)=\operatorname{div}\left(\frac{\lambda}{c_{p}} \operatorname{grad} T\right)+S_{h}
\end{aligned}
$$




\subsubsection{Governing equations}

The flow, mass transfer and heat transfer process of liquid helium and gas helium in the inner container of the liquid helium storage tank must follow the conservation of mass, the conservation of momentum and the conservation of energy. The governing equation is shown in Eq. (1).

The thermal conductivity of fluid is defined as Eq. (2), energy (E) is treated as mass-averaged variables by Eq.(3).

$$
\begin{aligned}
& \lambda=\alpha_{l} \lambda_{l}+\alpha_{v} \lambda_{v} \\
& E=\frac{\sum_{q=1}^{n} \alpha_{q} \rho_{q} E_{q}}{\sum_{q=1}^{n} \alpha_{q} \rho_{q}}
\end{aligned}
$$

For each phase, $\mathrm{Eq}(3)$ is calculated from its specific heat and temperature. For mass-averaged temperature, $\mathrm{E}$ is replaced with $\mathrm{T}$.

\subsubsection{Turbulence model}

According to the research results of previous scholars on the thermal response law of liquid hydrogen, the natural convection flow pattern along the heated wall is turbulent. Considering that the model of storage tank contains a curved wall surface, therefore, the Realizable $k-\varepsilon$ model considering wall surface curvature is selected, and the equation is shown in Eq.(4).

$$
\begin{aligned}
& \frac{\partial(\rho k)}{\partial t}+\frac{\partial\left(\rho k u_{j}\right)}{\partial x_{j}}=\frac{\partial}{\partial x_{j}} {\left[\left(\mu+\frac{\mu_{t}}{\sigma_{k}}\right) \frac{\partial k}{\partial x_{j}}\right]+G_{k}+G_{b}-\rho \varepsilon-Y_{M}+S_{k} } \\
& \frac{\partial(\rho \varepsilon)}{\partial t}+\frac{\partial\left(\rho \varepsilon u_{j}\right)}{\partial x_{j}}=\frac{\partial}{\partial x_{j}}\left[\left(\mu+\frac{\mu_{t}}{\sigma_{\varepsilon}}\right) \frac{\partial \varepsilon}{\partial x_{j}}\right]+C_{1 \varepsilon} \frac{\varepsilon}{k} C_{3 \varepsilon} G_{b}-\rho C_{2} \frac{\varepsilon^{2}}{k+\sqrt{v \varepsilon}} \\
&+\rho \varepsilon \sqrt{2 S_{i j} S_{i j}} \max \left[0.43, \frac{k \sqrt{2 S_{i j} S_{i j}}}{5 \varepsilon+k \sqrt{2 S_{i j} S_{i j}}}\right]+S_{\varepsilon}
\end{aligned}
$$

\subsubsection{Two-phase flow model}

Since there is a free liquid surface in the storage tank,the VOF model is selected as the two-phase flow model. The volume ratio equation is shown in Eq. (5)[6].

$$
\frac{1}{\rho_{q}}\left[\frac{\partial}{\partial t}\left(\alpha_{q} \rho_{q}\right)+\nabla \cdot\left(\alpha_{q} \rho_{q} \vec{v}_{q}\right)=\sum_{p=1}^{n}\left(\dot{m}_{p q}-\dot{m}_{q p}\right)\right] \quad \sum_{q=1}^{n} \alpha_{q}=1
$$


Mass or volume-averaged cell properties are used in a control volume containing more than one phases, as appropriate. For instance, viscosity and density, appearing in the transport equations are computed using the following volume-averaged equations.

$$
\begin{aligned}
& \rho=\alpha_{q} \rho_{q}+\left(1-\alpha_{q}\right) \rho_{q} \\
& \mu=\alpha_{q} \mu_{q}+\left(1-\alpha_{q}\right) \mu_{q}
\end{aligned}
$$

\subsection{Phase change model}

In this paper, the Lee model is used to describe the evaporation and condensation process of liquid helium [6]. The saturation temperature of liquid helium is selected from the data in reference and the function of saturation temperature with pressure is obtained by polynomial fitting[9].The Lee model is shown in Eq.(4).

$$
\begin{aligned}
& T_{l}>T_{s a t} \quad S_{m}=\dot{m}_{l v}=c \alpha_{l} \rho_{l}\left(\left(T_{l}-T_{\text {sat }}\right) / T_{\text {sat }}\right) \\
& T_{v}<T_{\text {sat }} \quad S_{m}=\dot{m}_{v l}=c \alpha_{v} \rho_{v}\left(\left(T_{\text {sat }}-T_{v}\right) / T_{\text {sat }}\right) \\
& S_{h}=S_{m} h_{f g}
\end{aligned}
$$

Table 1 shows the mass transfer intensity factors adopted by different scholars According to research results of Abdullah Saleem ${ }^{[6]}$, it has good adaptability to take the mass transfer intensity factor of 0.1.Therefore in this paper,the mass transfer intensity factor 0.1 is adopted.

Table 1. Application of mass transfer intensity factor

\begin{tabular}{c|c|c|c|c|c}
\hline Researchers & $\begin{array}{c}\text { De } \\
\text { Schepper }^{[10]}\end{array}$ & Wu $^{[11]}$ & Yang $^{[12]}$ & Goodson $^{[13]}$ & $\begin{array}{c}\text { Abdullah } \\
\text { Saleem }^{[6]}\end{array}$ \\
\hline $\begin{array}{c}\text { Mass transfer intensity } \\
\text { factor }\end{array}$ & 0.1 & 0.1 & 100 & 100 & $\begin{array}{c}0.1 \text { (Good } \\
\text { adaptability) }\end{array}$ \\
\hline
\end{tabular}

\subsection{Boussinesq equation}

The flow in the LHe tank is natural convection, through using Boussinesq approximation, a faster convergence can be achieved. This approximation treats density as constant except the buoyancy term in the momentum equation. The essence of this assumption is neglecting the effect of density variation on the flow field, except for the buoyancy term. With this approximation, computational cost, as well as the nonlinearity of the problem, can be reduced significantly. The Boussinesq equation is given by Eq.(8).

$$
\left(\rho-\rho_{0}\right) g \approx-\rho_{0} \beta\left(T-T_{0}\right) g
$$

The Boussinesq approximation is valid for small density changes., $\beta\left(T-T_{0}\right) \ll 1$. In our case, we use Boussinesq approximation for liquid helium density change only.

\subsection{Initial and Boundary conditions}


The initial velocity of the fluid in the computational domain is $0 \mathrm{~m} / \mathrm{s}$, the initial temperature of the fluid is $4.2 \mathrm{~K}$, and the initial pressure of the gas is $96000 \mathrm{~Pa}$.

Due to the low temperature of liquid helium, slight temperature changes in different parts of the inner container of the storage tank have little effect on heat leakage. Therefore, it is assumed that the boundary condition of the wall is the wall boundary with uniform heat flux without slip. In order to quantitatively analyze the influence of evaporation rate on the thermal response process of the storage tank, five conditions of evaporation rate are set for analysis. The lower evaporation rate is $0.484 \%$, which is calculated byEq(9)[7], and the upper evaporation rate is $0.692 \%$,which is obtained through standards[8]. Heat leakage can be calculated by Eq.(10) the calculated heat leakage is shown in Table 2.

$$
\begin{aligned}
& q=q_{r}+q_{g}+q_{s}=K \frac{T_{h}-T_{c}}{\delta}=\left(K_{r}+K_{g}+K_{s}\right) \frac{T_{h}-T_{c}}{\delta} \\
& K_{r}=\frac{1}{N} \frac{\sigma\left(T_{h}+T_{c}\right)\left(T_{h}^{2}+T_{c}^{2}\right)}{\delta_{s}(\alpha+2 \gamma) / 2 n+1 / \varepsilon_{1}+1 / \varepsilon_{1}-1} \\
& K_{g}=\frac{\lambda_{p}}{2 \beta l_{0}(n+1) / \delta} p_{0} \\
& K_{s}=a N^{2}\left(T_{h}+T_{c}\right) / 2+b\left[\left(T_{h}+T_{c}\right)\left(T_{h}^{2}+T_{c}^{2}\right)\right] / N \\
& \alpha=\frac{24 \times Q}{\rho V \gamma} \times 100 \%
\end{aligned}
$$

Since the filling rate is a major factor affecting the thermal response process of cryogenic storage tanks, it is necessary to study the influence of the filling rate on the thermal response process of storage tanks. The inner container of the liquid helium storage tank needs to reserve $5 \%-10 \%$ of the volume for gas-phase storage. In order to study the influence of initial filling rate on the pressure rise and temperature rise law of the inner container of the storage tank, the liquid filling rate at the initial time is calculated under five working conditions of $90 \%, 89 \%, 87.5 \%, 86 \%, 85 \%$, and $84 \%$. The liquid level heights at different filling rate can be calculatied by Eq(11), the calculated liquid level heights are shown in Table 2.

$$
\begin{aligned}
V & =V_{l}+V_{f}=\frac{1}{2} \pi R^{2} L+\int_{0}^{V_{0}} 2 \sqrt{R^{2}-t^{2}} L d t \\
& =\frac{1}{2} \pi R^{2} L+2 L\left(\frac{1}{2} Y_{0} \sqrt{R^{2}-Y_{0}^{2}}+\frac{1}{2} R^{2} \arcsin \frac{Y_{0}}{R}\right)+\frac{\pi}{18} D^{3}+\frac{9}{4} \pi\left(h^{2} Y_{0}-\frac{1}{3} Y_{0}^{3}\right)
\end{aligned}
$$

Table 2 Heat leakage of the container and level of the liquid helium

\begin{tabular}{c|c|c|c|c|c|c}
\hline Evaporation rate/\% & 0.692 & 0.645 & 0.605 & 0.565 & 0.525 & 0.484 \\
\hline Heat leakage/W & 75.002 & 69.902 & 65.567 & 61.232 & 56.897 & 52.454 \\
\hline Filling rate/\% & 0.9 & 0.89 & 0.875 & 0.86 & 0.85 & 0.84 \\
\hline Liquid level height/m & 0.938 & 0.903 & 0.855 & 0.809 & 0.779 & 0.754 \\
\hline
\end{tabular}




\section{Numerical implementation}

The pressure-based unsteady numerical simulation method in Fluent software is adopted. And Turbulence model, multiphase flow model, and Lee model are set in Fluent software. The whole calculation process meets the equations of mass conservation, momentum conservation, and energy conservation. The continuity equation, momentum equation, and energy equation all adopt the second-order upwind scheme and the pressure-velocity coupling algorithm adopts PISO algorithm. Considering the actual change process of the physical parameters of liquid helium and gas helium, the density and other physical parameters of liquid helium and gas helium take the values in references[9]and[14], so as to add the function of physical parameters on temperature to the material library through polynomial fitting[9,14]. In addition, the surface tension of the liquid surface of the cryogenic storage tank has been studied by using CSF model. Only in microgravity can surface tension affect thermal stratification of cryogenic liquid $[15,16]$. Therefore, the influence of the surface tension will not be considered. Fig.3 shows flowchart of numerical simulation.

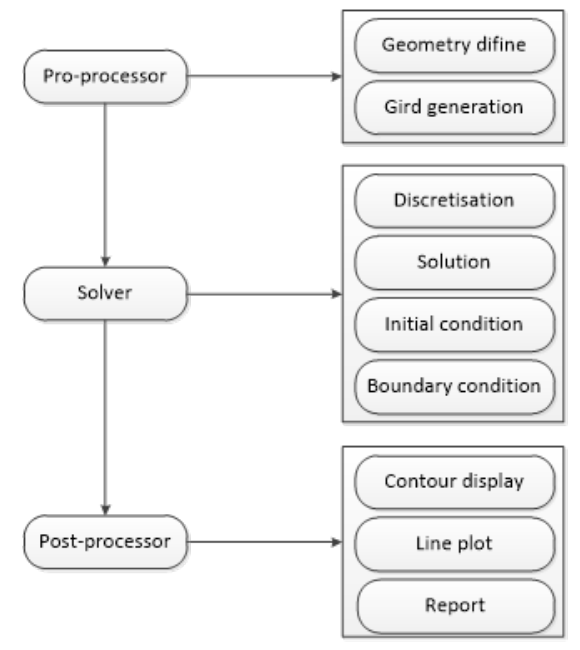

Fig.3 Flowchart of numerical simulation

In this paper, Fluent was used to simulate the thermal response process of liquid helium tank under different evaporation rates and filling rates within 100 minutes.

\section{Numerical implementation}

\subsection{Grid independence study}

ICEM is used to divide the computing domain into O-shaped structured grids and the grids near the wall are encrypted. Fig. 4 shows the grid used for CFD simulation. 


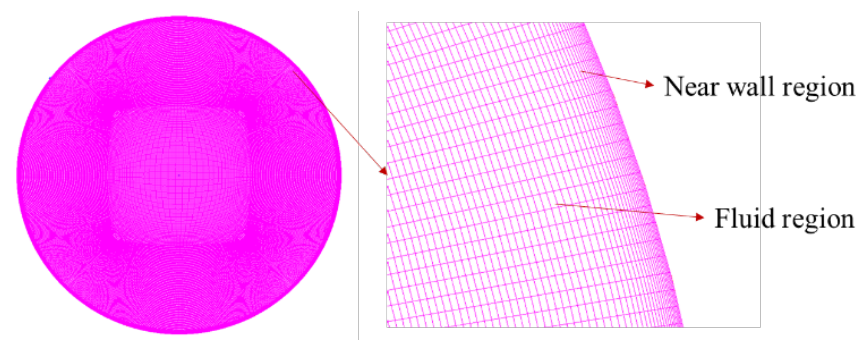

Fig.4 Grid used for CFD simulation

Fig. 5 shows the results of grid independence verification. Based on the initial and boundary conditions and coupling algorithm showed above, the CFD model is calculated for 100min under grid 41085, 83205, 151380, 198005 respectively. The average temperature of through line $(X=0, Y>Y 0)$ in gas zone is obtained by report feature of Fluent, It can be seen that when the number of grids is increased from 151380 to 198005, there is no obvious change in the results. Therefore, the grid 151380 is adopted for calculation in this paper.

\subsection{Validation of CFD model}

Temperature distribution tested by Bora M.S [4] is used to validate the current CFD model. When the simulation reaches steady state, the average temperature of through line $(\mathrm{X}=0, \mathrm{Y}>\mathrm{Y} 0)$ in gas zone is taken as the basis for verification, which is obtained by plot feature of Fluent.Fig. 6 displays the comparison of the experiment result and the numerical simulation result.

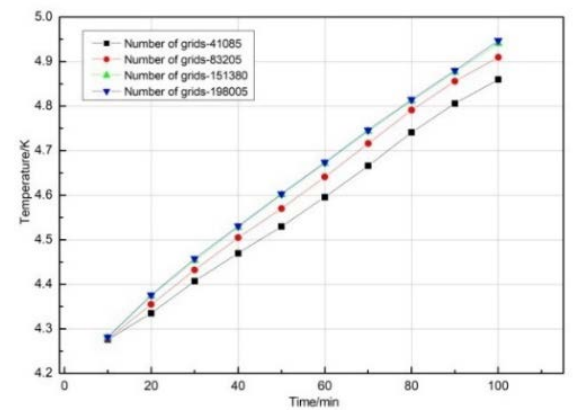

Fig.5 Grid-independent validation

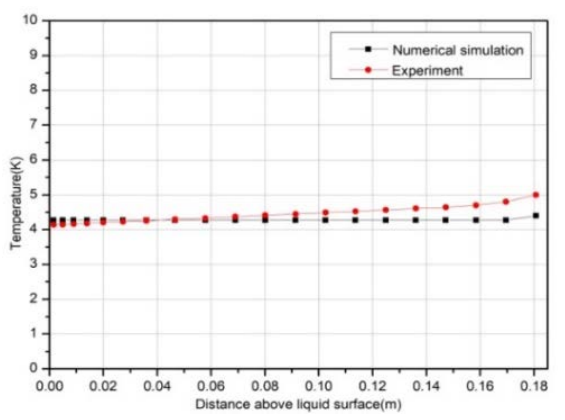

Fig.6 Temperature comparison of the gas field 
The comparison shows that the temperature above the free surface is more uniform than the experimental value, and the trend of the two is consistent. Therefore, the model can be used to predict the thermal response of the liquid helium tank.

\section{Numerical implementation}

\subsection{Thermal stratification}

Tecplot is used to plot the temperature field with the result calculated by Fluent. Fig. 7 shows the temperature contours of the flow field when the filling rate is $90 \%$ and the evaporation rate is $0.484 \%$ for $30 \mathrm{~min}$, 50min, $70 \mathrm{~min}$, and $90 \mathrm{~min}$.It can be seen that thermal stratification occurs in the liquid helium storage tank during the thermal response process and the temperature gradient in the gas phase is larger than that in the liquid phase. With the continuous entry of heat, the temperature of gas-phase gradually rises. On both sides of the gas-liquid interface near the wall surface, heat is provided by liquid helium rising along the wall surface on the one hand, and heat flow coming in from the wall surface of the inner container on the other hand. As a result, the temperature on both sides is higher than that in the middle. At the same time, gas helium with slightly higher temperature transfers heat to liquid helium, so the temperature from the wall surface to the middle position on the same isotherm shows a change rule from small to large and then from large to small with a higher temperature area in the middle. In addition, with the increase of simulation time, temperature stratification has gradually formed. For example, the central part of the isotherm has a larger protrusion at $50 \mathrm{~min}$ and $70 \mathrm{~min}$, while the central part of the isotherm is very flat at $100 \mathrm{~min}$, which is because heat continuously enters the main flow region of liquid helium through convection and conduction.

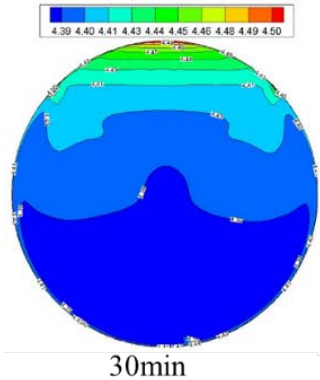

$30 \mathrm{~min}$

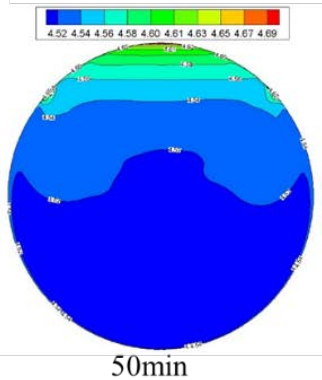

$50 \mathrm{~min}$

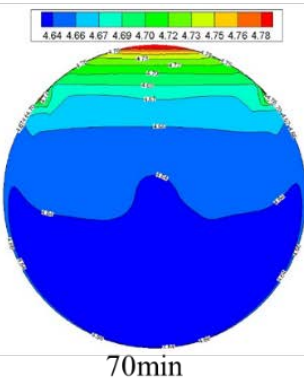

$70 \mathrm{~min}$

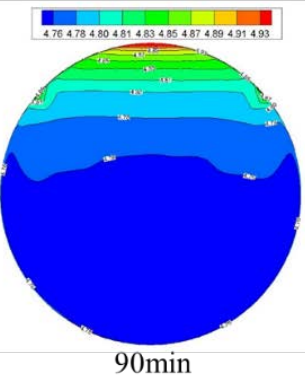

$90 \mathrm{~min}$

Fig. 7 Temperature distribution of flowfield

The 'plot' feature of Fluent is used to get the temperature of gas phase and free liquid surface. In order to ensure the accuracy of the measurement results, measurements were taken along the pre-defined path line $\mathrm{X}=0, \mathrm{Y}>\mathrm{Y} 0$ and $\mathrm{Y}=\mathrm{Y} 0$. Fig. 8 shows the temperature variation curve of the gas phaseof the storage tank and Fig. 9 shows the temperature variation curve of the free liquid surfaceof the storage tank. It can be seen that the temperature shows a gradual distribution in the vertical direction and the temperature gradient is small. At the same time, the helium gas at the top of the gas phase is slightly higher than the helium gas below it due to the influence of wall heat leakage. On the free liquid surface, the temperature of liquid helium near the wall surface is significantly higher than the temperature of liquid helium inside. The temperature gradient near the wall is large and a thermal boundary layer appears. With the progress of the simulation process, the free surface temperature increases. For example, the free surface temperature is about $4.55 \mathrm{~K}$ for $50 \mathrm{~min}$ and $4.8 \mathrm{~K}$ for $100 \mathrm{~min}$. 


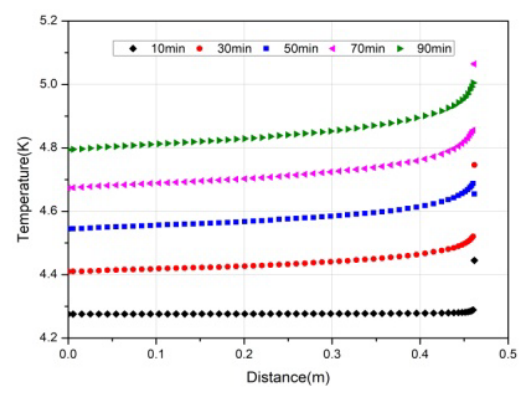

Fig.8 Temperature curve of the gas phase.

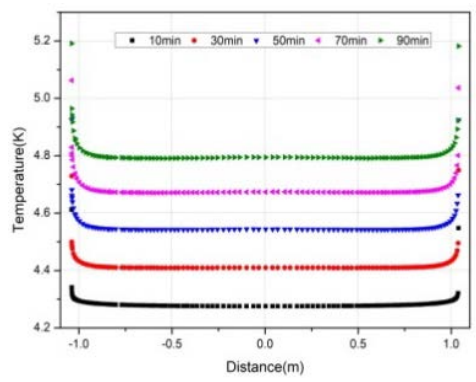

Fig.9 Temperature curve of free surface

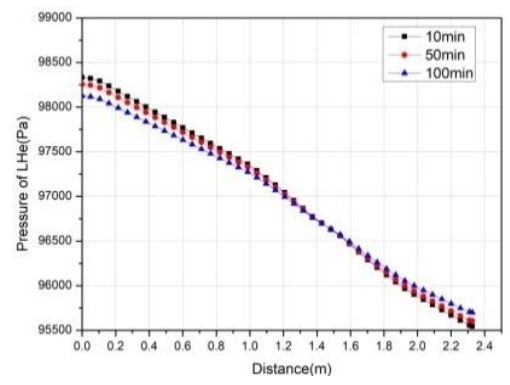

Fig.10 Pressure curve of the liquid phase

\subsection{Pressure distribution}

Fig. 11 shows the pressure contours of the storage tank when the filling rate is $90 \%$,the evaporation rate is $0.484 \%$ for $10 \mathrm{~min}, 50 \mathrm{~min}$ and $100 \mathrm{~min}$. It can be seen that the gas phase pressure has been around $95500 \mathrm{~Pa}$. However, in the liquid phase, the pressure distribution in the tank shows a stepped distribution rule with a lower level and a higher level due to static pressure. The pressure on the bottom liquid is the largest, which requires high strength on the tank bottom. Same method as measuring temperature, the pressure in the liquid region was measured. Fig. 10 shows the pressure curve in the liquid helium $(X=0, Y<Y 0)$. Comparing the curves of Fig. 10, it is found that the pressure in the liquid helium region is approximately linearly distributed. With the increase of simulation time, the pressure at the bottom of the liquid helium decreases slightly, which is because heat gradually enters the liquid helium, warming up the liquid helium slowly, the density gradually decrease. However, the pressure in the top of the liquid helium is slightly increasing, which may be caused by 
the liquid helium flowing toward the centre of the liquid surface after rising along with the natural convection on the wall.
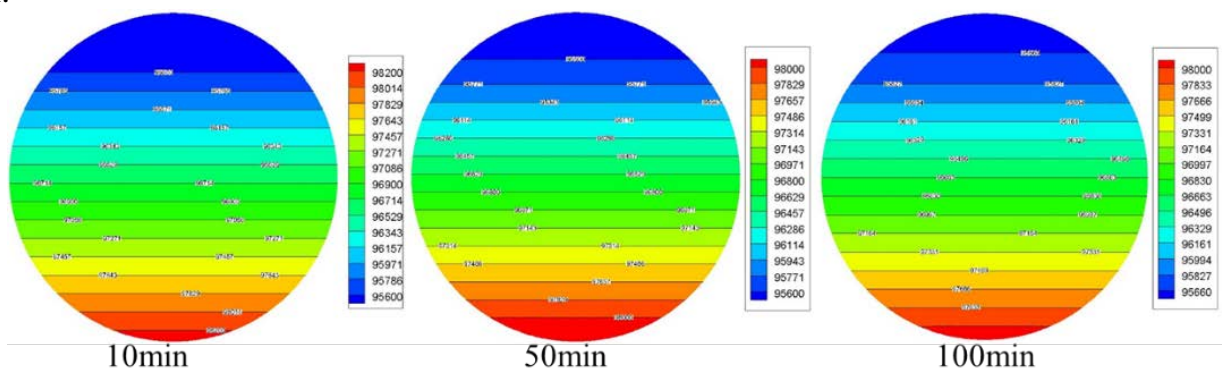

Fig. 11 Pressure distribution of flowfield

\subsection{Velocity distribution}

Fig. 12 shows the velocity contour at $10 \mathrm{~min}, 50 \mathrm{~min}$ and the velocity vector diagram at $20 \mathrm{~min}, 100 \mathrm{~min}$ when filling rate is $90 \%$, evaporation rate is $0.484 \%$. Fig. 12 shows the vector diagram of the velocity in the liquid phase, the gas phase and the middle of the liquid phase near the free liquid surface of the tank at 100 min under this condition.
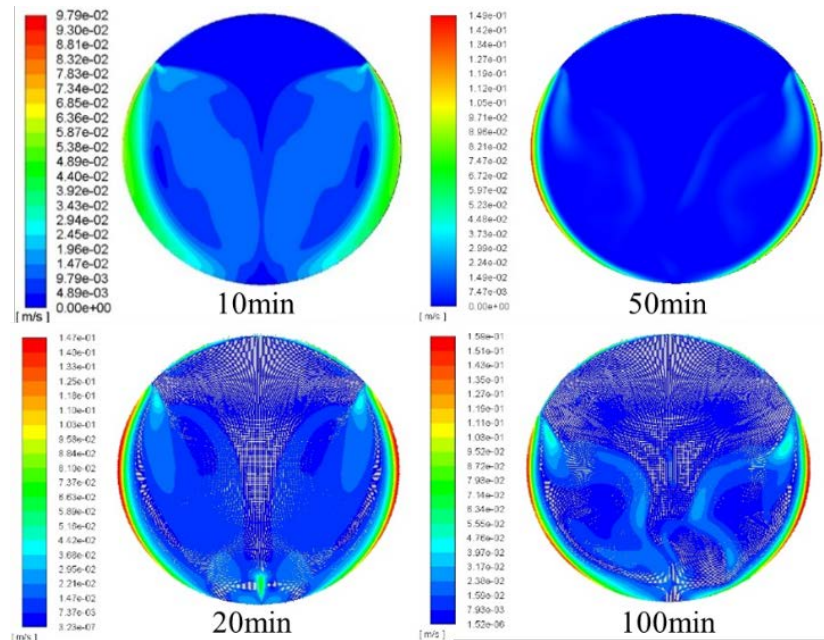

Fig. 12 Velocity diagram and velocity vector diagram of flowfield
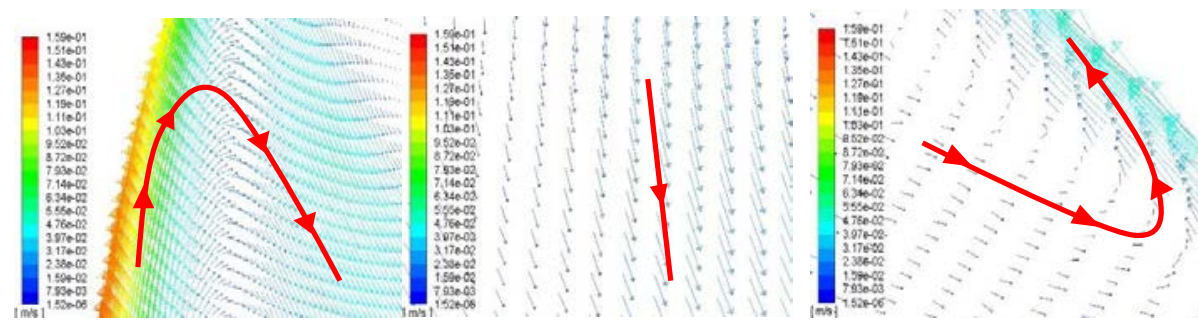

Fig. 13 Velocity vector diagram near the liquid surface (left), gas phase (right) and middle of the liquid phase (middle) 
As shown in Fig. 13 (left), it is found that the density of liquid helium at the wall surface decreases after being heated, thus generating natural convection flowing upward along the wall surface by the action of buoyancy force and finally flowing into the liquid helium core area after rising to the free liquid surface. Using the improved Rayleigh number, the $\mathrm{Ra}^{*}$ of natural convection is calculated to be larger than the critical value 1011, so the natural convection flow pattern is turbulent. As the temperature of the flow field rises, the buoyancy force is insufficient to maintain the liquid helium flowing to the free liquid surface. At the same time, with the increase of simulation time, heat continuously enters the liquid phase, making the timing for convective liquid helium to flow out of the wall surface and into the liquid helium core area continuously advance. As shown in Fig. 13 (middle), the part of liquid helium flows to the bottom of the storage tank after flowing into the liquid helium core area to compensate for the energy of the liquid helium. Moreover, helium convection also exists in the gas phase region. As shown in Fig. 13 (right), helium near the wall surface flows upward along the wall surface after being heated, while helium at the upper part of the gas phase flows downward to supplement helium at the bottom of the gas phase. Due to the convection of liquid helium and gas helium, convection circulation occurs in the gas phase and the liquid phase.

In order to study the natural convection process clearly, the 'pathlines' feature of Fluent is used to track the trace of natural convection. Fig. 14 shows the flow path diagram of the flow field for $20 \mathrm{~min}$, 40min, 60min, $80 \mathrm{~min}$ and $100 \mathrm{~min}$, among them, each diagram is composed of two parts, the pathline diagram is in left and the phase diagram is in right. Obviously, there is natural convection cycle in LHe tank.
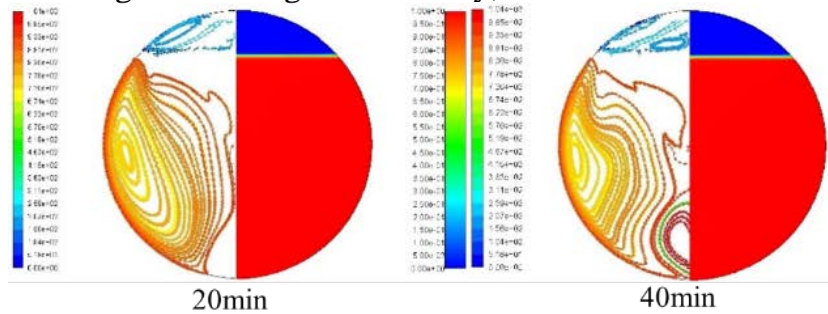

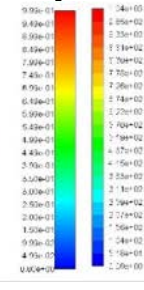

$40 \mathrm{~min}$

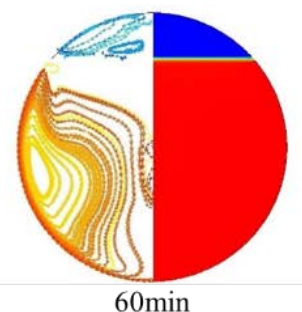

$60 \mathrm{~min}$
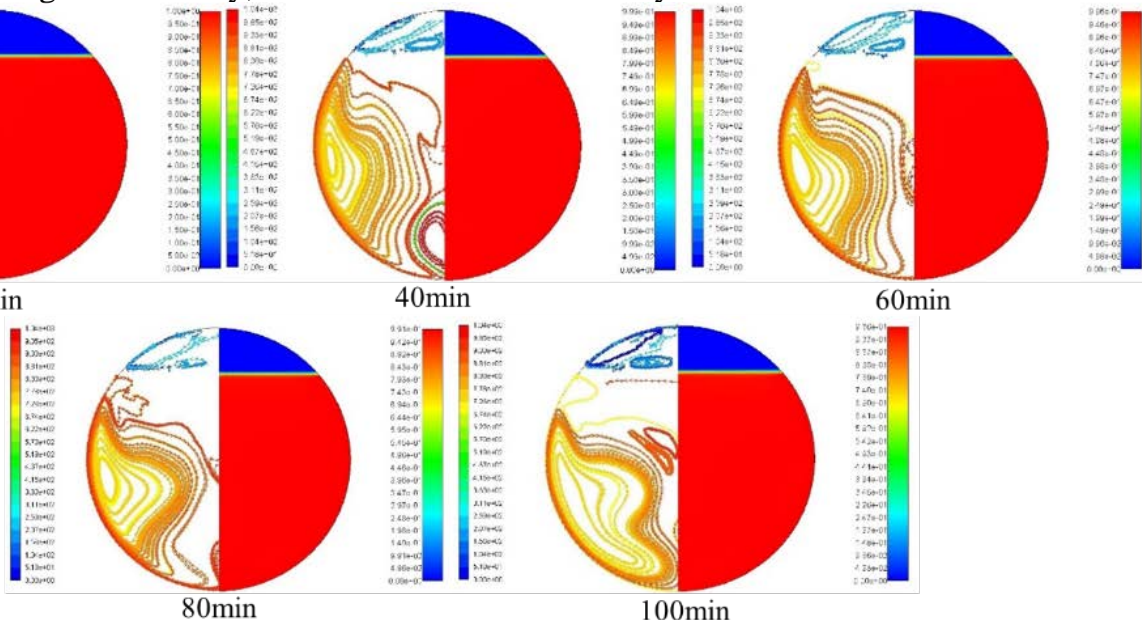

$100 \mathrm{~min}$

Fig. 14 Pathline diagram of flowfield

At the beginning of the simulation, the convection circulation in the gas and liquid zone is stable. However, with the increase of the simulation time, the circulation in the gas phase and liquid phase is gradually unstable and even secondary circulation occurs in the liquid helium core region and gas helium core region.

\subsection{Analysis of temperature and pressure curves}

Fig.15 shows the temperature and pressure curves of helium gas at different evaporation rates when the filling rate is $90 \%$. Fig. 16 shows the temperature and pressure curves of helium gas at different filling rates when the evaporation rate is $0.484 \%$. 

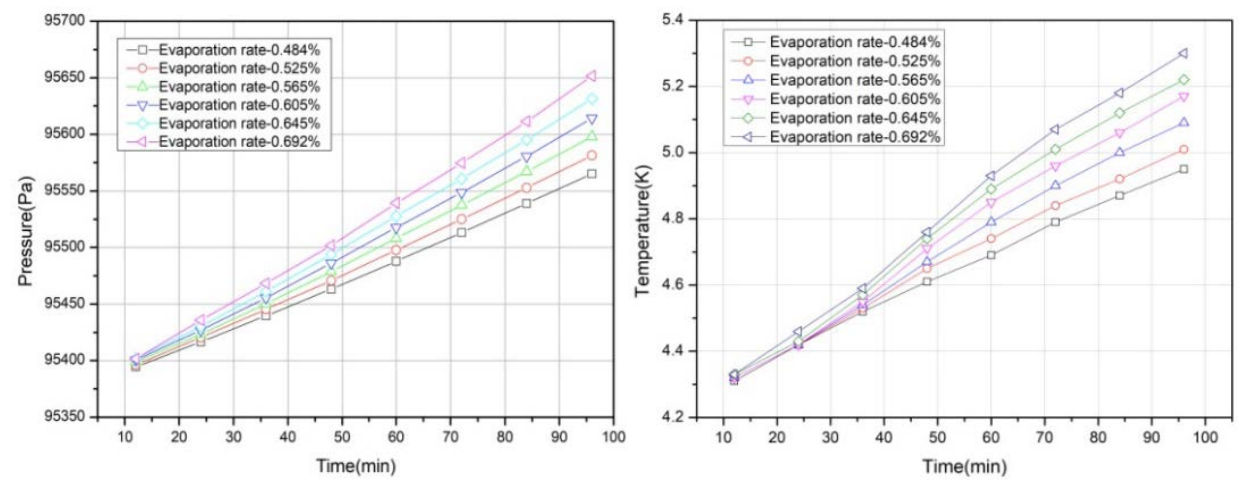

Fig.15 The pressure and temperature curve of GHe at different evaporation rates

Comparing the temperature curve and pressure curve at different evaporation rates, it can be seen that with the increase of simulation time, the pressure and temperature of the gas phase in the storage tank increase approximately linearly. The higher the evaporation rate, the higher the increments in the velocity of pressure and temperature of the gas phase of the storage tank. Therefore, improving the thermal insulation performance of the storage tank is the key to the safe storage and transportation of liquid helium.
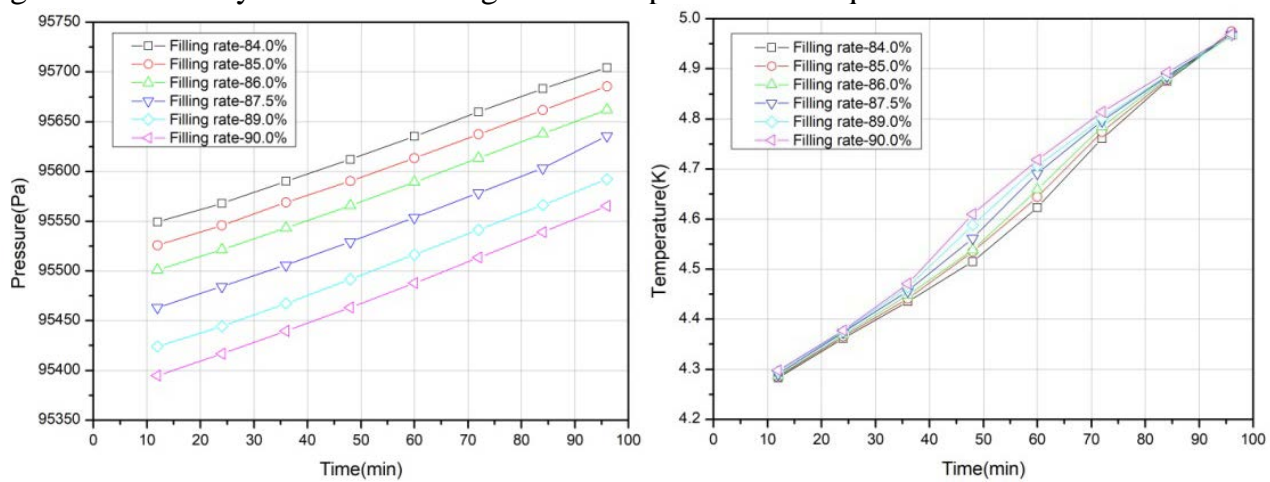

Fig.16 The pressure and temperature curve of GHe at different filling rates

Comparing the pressure curve and temperature curve at different filling rates, it can be seen that the pressure of the gas phase increases linearly. The higher the filling rate, the lower the pressure in the gas phase. But the increments in the velocity of pressure at different filling rate are similar, which may be due to the lower free surface area of liquid helium as the filling rate increases. The temperature of the gas phase increases approximately linearly with the increase of the simulation time. In the process of changing the filling rate from $84 \%$ to $90 \%$,, the temperature curve of the gas phase in the liquid helium storage tank changes obviously only when the simulation time is about $60 \mathrm{~min}$, which indicates that the filling rate has little influence on the temperature law of the gas phase in the storage tank.

\section{Conclusions}

According to the thermal response process of the $85 \mathrm{~m}^{3}$ storage tank of railway tanker filled with liquid helium, the thermal response law of the tank is studied by the numerical method under the premise of 
considering the mass transfer and heat transfer between phases. The influence of evaporation rate and filling rate on the pressure and temperature law of the tank is discussed and the following conclusions are obtained:

(1) With the continuous leakage of heat, liquid helium in the tank gradually develops thermal stratification. And the isotherm gradually flattens with the increase of simulation time. Due to the existence of static pressure, the pressure of liquid helium has a stepped distribution with small upper part and large lower part, while the pressure of gas helium has a uniform distribution. With the increase of simulation time, the pressure in the upper part of liquid helium increases slowly, while the pressure in the lower part of liquid helium decreases slowly.

(2) After the tank is heated, the temperature near the wall rises, causing the density to decrease, thus slowly rising to the free liquid surface along the wall by the action of buoyancy force, generating turbulent natural convection. The longer the simulation time, the earlier the rising liquid helium near the wall will flow into the liquid helium.

(3) The higher the evaporation rate of the storage tank, the higher the increments in the velocity of pressure and temperature in the tank. The larger the filling rate, the smaller the initial pressure in the gas phase of the storage tank. Because of the larger the filling rate, the smaller the free liquid surface of liquid helium in the storage tank.

The above research and conclusion have important value to clarify the temperature and pressure rising principle of railway liquid helium storage tanker.

\section{References}

[1] Yi Shen, Ning Jiang, et al., 2001. Storage and Emission of Supercritical Helium. Int. J. Cryogenics 4, 48-53.

[2] Man Man, Liqiang Zhang, et al. Experimental Study and Heat Leakage Analysis of Supercritical Helium Storage Tank. Int. J. Cryogenics, 2013 33-37.

[3] R.T. Swim, 1960. Temperature Distribution in Liquid and Vapor Phases of Helium in Cylindrical Dewars. Int, J. Advances in Cryogenic Engineering 7, 498-503.

[4] Bora M.S, Rugaiganisa, et al., 1990. Experimental investigation on heat leak into a liquid helium dewar. Int, J. Cryogenics 30, 942-946.

[5] Stephen G, Sydoriak et al., 1951. Low Evaporation Rate Storage Vessel for Liquid Helium. Int, J. Review of Scientific Instruments 22, 915-919.

[6] John D.Anderson,JR.Computational Fluid Dynamics-The Basics with Applications.1992

[7] Abdullah Saleem, Shamsuzzaman Farooq, et al., 2018. A CFD simulation study of boiling mechanism and BOG generation in a full-scale LNG storage tank. Int, J. Computers and Chemical Engineering 115,112-120.

[8] Cunnington G R,Tien C L.A study of heat -transfer process in multiplayer insulation. Progress in Astronautics and Aeronautics,N.Y.:Academic Press,1970(23):111-126.

[9] GB 18442-2001,Cryo-insulaiton pressure vessels.2001.

[10] Guobang Chen, RuiBao, 2006. Cryogenic Engineering Technology, Data Volume. Int, M. Chemical Industry Press Co, Lid, 99-120. 
[11] De Schepper, S.C., et al., 2009. Modeling the Evaporation of a Hydrocarbon Feedstock in the Convection Section of a Steam Cracker. Int, J. Comput. Chem. Eng. 33,122-132.

[12] Wu, H., et al., 2007. Simulation of Refrigerant Flow Boiling in Serpentine Tubes. Int, J. Heat Mass Transfer 50, 1186-1195.

[13] Yang, Z., et al., 2008. Numerical and Experimental Investigation of Two-phase Flow during Boiling in a Coiled Tube. Int, J. Heat Mass Transfer 51, 1003-1016.

[14] Goodson, K., et al., 2010. The volume of fluid simulation of boiling two-phase flow in a vapor-venting microchannel. Int, J. Front. Heat Mass Transf. (FHMT) 1, 1-11.

[15] Vincent D, Arp Robert D, et al., 1989. Thermophysical Properties of Helium-4 From 0.8 to $1500 \mathrm{~K}$ With Pressures to 2000 MPa. Int, M. National Institute of Standards and Technology Technical Note 1334.

[16] Zhan Liu, Cui Li, 2018. Influence of slosh baffles on thermodynamic performance in liquid hydrogen tank. Int, J. Journal of Hazardous Materials 346, 253-262.

[17] Juan Fu, Bengt Sunden, 2013. Analysis of Self-Pressurization Phenomenon in a Cryogenic Fluid Storage Tank with VOF Method. Proceedings of the ASME 2013 International Mechanical Engineering Congress and Exposition, USA

\section{Appendix}

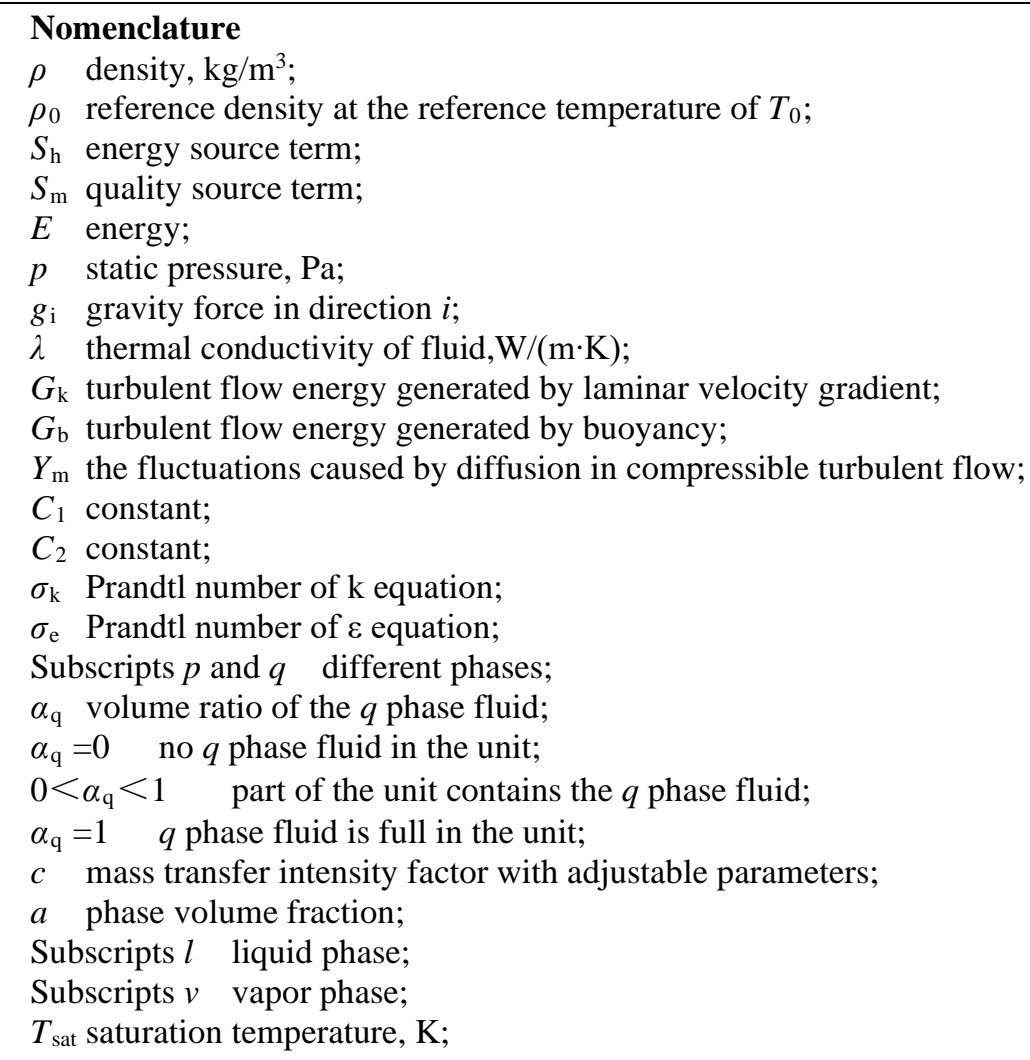




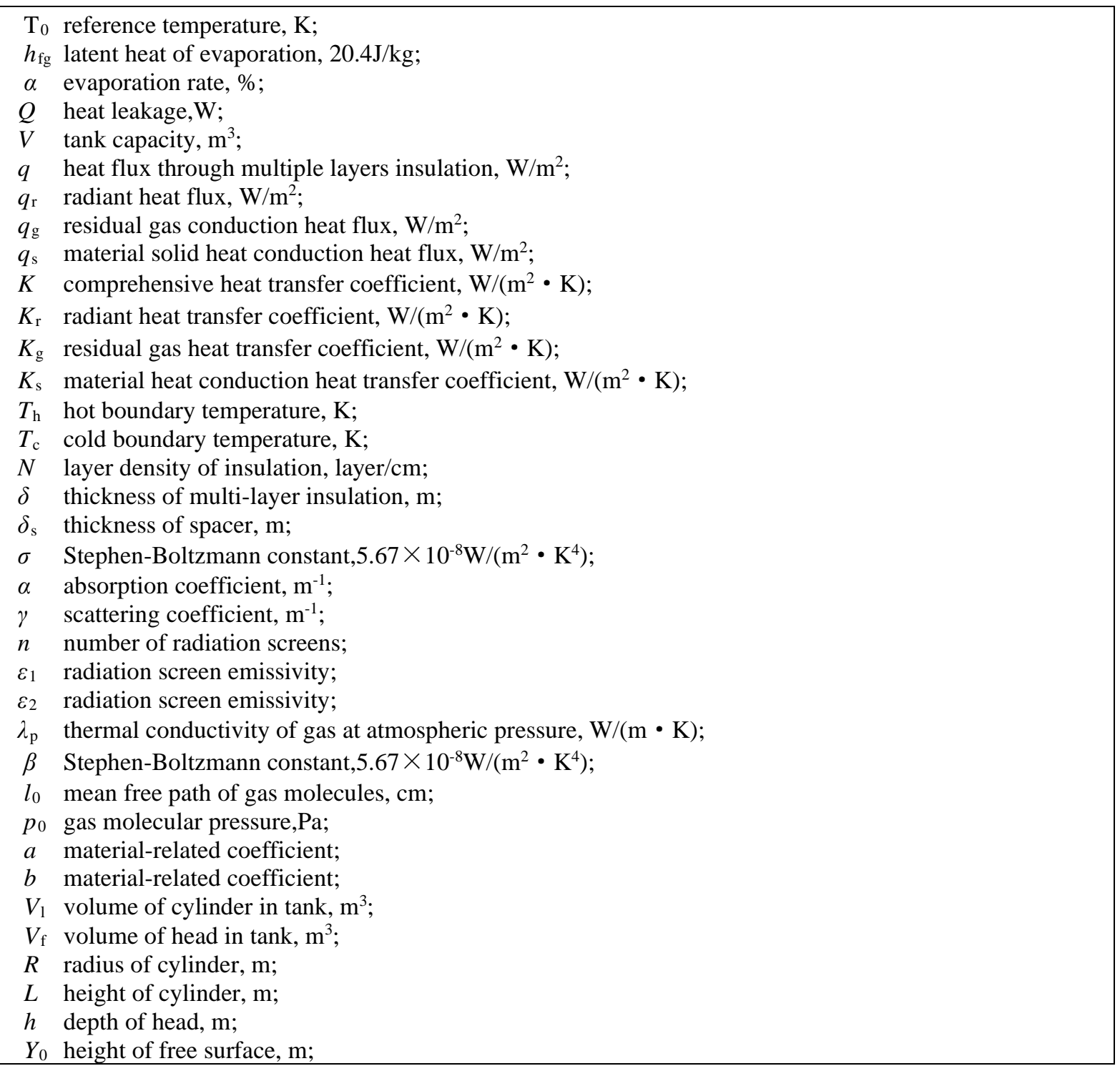




\section{Authors' Profiles}

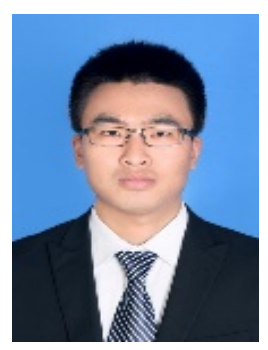

Wang Yuzhu, born in 1989, male, he is a engineer with master degree, graduated from University of Petroleum in Chin. He is engaged in design and management.

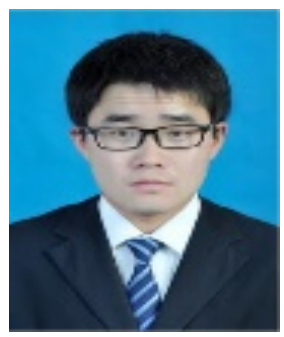

Zhang Caigong, born in 1992, male, he is from China.and he is a postgraduate.

How to cite this paper: Wang Yuzhu, Cui Lande, Zhang Caigong, Kong Lingfen, Jia Wenlong. "CFD Investigation of Thermal and Pressurization Performation in LHe Tank", International Journal of Engineering and Manufacturing(IJEM), Vol.10, No.1, pp.12-28, 2020. DOI: 10.5815/ijem.2020.01.02 